Kroll, Frank-Lothar

\title{
Möglichkeiten und Notwendigkeiten historiographischen Arbeitens in der
} Berufs- und Wirtschaftspädagogik

Seifried, Jürgen [Hrsg.]; Faßhauer, Uwe [Hrsg.]; Seeber, Susan [Hrsg.]: Jahrbuch der berufs- und wirtschaftspädagogischen Forschung 2014. Opladen [u.a.] : Verlag Barbara Budrich 2014, S. 11-19. (Schriftenreihe der Sektion Berufs- und Wirtschaftspädagogik der Deutschen Gesellschaft für Erziehungswissenschaft (DGfE); 217)

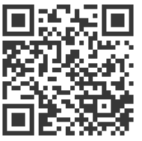

Quellenangabe/ Reference:

Kroll, Frank-Lothar: Möglichkeiten und Notwendigkeiten historiographischen Arbeitens in der Berufsund Wirtschaftspädagogik - In: Seifried, Jürgen [Hrsg.]; Faßhauer, Uwe [Hrsg.]; Seeber, Susan [Hrsg.]: Jahrbuch der berufs- und wirtschaftspädagogischen Forschung 2014. Opladen [u.a.] : Verlag Barbara Budrich 2014, S. 11-19 - URN: urn:nbn:de:0111-pedocs-97220 - DOI: 10.25656/01:9722

https://nbn-resolving.org/urn:nbn:de:0111-pedocs-97220

https://doi.org/10.25656/01:9722

in Kooperation mit / in cooperation with:

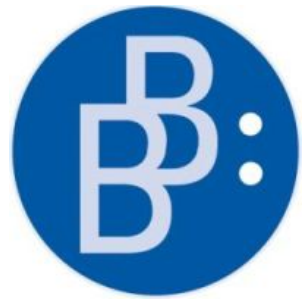

https://www.budrich.de

\section{Nutzungsbedingungen}

Dieses Dokument steht unter folgender Creative Commons-Lizenz:

http://creativecommons.org/licenses/by-nc-nd/3.0/de/deed - Sie dürfen das Werk bzw. den Inhalt unter folgenden Bedingungen vervielfältigen, verbreiten und öffentlich zugänglich machen: Sie müssen den Namen des Autors/Rechteinhabers in der von ihm festgelegten Weise nennen. Dieses Werk bzw. dieser Inhalt darf nicht für kommerzielle Zwecke verwendet werden und es darf nicht bearbeitet, abgewandelt oder in anderer Weise verändert werden.

Mit der Verwendung dieses Dokuments erkennen Sie die Nutzungsbedingungen an.

\section{Terms of use}

This document is published under following Creative Commons-License: http://creativecommons.org/licenses/by-nc-nd/3.0/de/deed.en - You may copy, distribute and transmit, adapt or exhibit the work in the public as long as you attribute the work in the manner specified by the author or licensor. You are not allowed to make commercial use of the work or its contents. You are not allowed to alter, transform, or change this work in any other way.

By using this particular document, you accept the above-stated conditions of use.

\section{Kontakt / Contact:}

\section{peDOCs}

DIPF | Leibniz-Institut für Bildungsforschung und Bildungsinformation Informationszentrum (IZ) Bildung

E-Mail: pedocs@dipf.de

Internet: www.pedocs.de

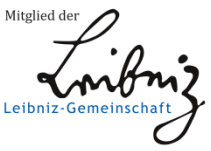




\section{Jahrbuch der berufs- und wirtschaftspädagogischen Forschung 2014}

Jürgen Seifried, Uwe Faßhauer Susan Seeber (Hrsg.)

DGE Deutsche Gesellschaft 
Schriftenreihe der Sektion

Berufs- und Wirtschaftspädagogik der Deutschen Gesellschaft für Erziehungswissenschaft (DGfE) 
Jürgen Seifried

Uwe Faßhauer

Susan Seeber (Hrsg.)

\section{Jahrbuch der berufs- und wirtschaftspädagogischen Forschung 2014}

Verlag Barbara Budrich

Opladen • Berlin • Toronto 2014 
Bibliografische Information der Deutschen Nationalbibliothek

Die Deutsche Nationalbibliothek verzeichnet diese Publikation in der Deutschen Nationalbibliografie; detaillierte bibliografische Daten sind im Internet über http://dnb.d-nb.de abrufbar.

(C) Dieses Werk ist im Verlag Barbara Budrich erschienen und steht unter folgender Creative Commons Lizenz: http://creativecommons.org/licenses/by-nc-nd/3.0/de/ Verbreitung, Speicherung und Vervielfältigung erlaubt, kommerzielle Nutzung und Veränderung nur mit Genehmigung des Verlags Barbara Budrich.

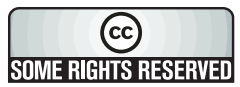

Dieses Buch steht im OpenAccess Bereich der Verlagsseite zum kostenlosen Download bereit (http://dx.doi.org/10.3224/84740164)

Eine kostenpflichtige Druckversion (Printing on Demand) kann über den Verlag bezogen werden. Die Seitenzahlen in der Druck- und Onlineversion sind identisch.

$$
\begin{array}{ll}
\text { ISBN } & 978-3-8474-0164-3 \text { (Paperback) } \\
\text { eISBN } & \mathbf{9 7 8 - 3 - 8 4 7 4 - 0 4 4 1 - 5 ~ ( e B o o k ) ~} \\
\text { DOI } & 10.3224 / 84740164
\end{array}
$$

Umschlaggestaltung: bettina lehfeldt graphic design, Kleinmachnow

Typografisches Lektorat: Judith Henning, Hamburg

Verlag Barbara Budrich, http://www.budrich-verlag.de 


\section{Inhaltsverzeichnis}

Vorwort 9

\section{Teil I: Perspektiven der historischen Berufsbildungsforschung}

Frank-Lothar Kroll

Möglichkeiten und Notwendigkeiten historiographischen Arbeitens in der Berufs- und Wirtschaftspädagogik........................................................ 1

Volker Bank, Annekathrin Lehmann

Theodor Franke. Sächsischer Pionier wirtschaftspädagogischen

Denkens in Deutschland

Marcel Schweder

Lehrerarbeit im Strafvollzug - Ein Diskurs aus historischer Sicht

\section{Teil II: Kompetenzmodellierung, -messung und -förderung}

Eveline Wittmann, Ulrike Weyland, Annette Nauerth, Ottmar Döring, Simone Rechenbach, Julia Simon, Iberé Worofka Kompetenzerfassung in der Pflege älterer Menschen - Theoretische und domänenspezifische Anforderungen der Aufgabenmodellierung 53

Simon Heinen, Martin Frenz, Christopher M. Schlick

Bildung für nachhaltige Entwicklung in der Gebäudeenergieberatung - Entwicklung eines Kompetenzmodells für die Förderung reflexiver Handlungsfähigkeit

Diana Stuckatz, Cornelia Wagner

Qualifizierungsangebote in der Pflegehilfe für Personen mit geringen Grundbildungskenntnissen - Empirische Studien zur Entwicklung von Lehr-Lern-Umgebungen und Arbeitsmaterialien. 81 


\section{Teil III: Gestaltung und Analyse von Lehr-Lern-Prozessen}

Eva Höpfer, Andrea Reichmuth, Doreen Holtsch, Franz Eberle

Wer sieht was? - Zum Umgang mit unterschiedlichen Sichtweisen auf

Unterricht am Beispiel des kaufmännischen Berufsschulunterrichts 95

Mandy Hommel

Sozial geteilte Reflexion - eine explorative Studie im

Mathematikunterricht. 109

Gerhard Minnameier, Rico Hermkes

„Kognitive Aktivierung“ und „konstruktive Unterstützung“ als Lehr-

Lern-Prozess-Größen - Eine Konzeption im rechnungswesen-

didaktischen Kontext

\section{Teil IV: Lehrerbildung und pädagogische Professionalität}

Nicole Kimmelmann, Johannes Lang

Lehramtsstudierende mit Migrationshintergrund und ihre

Schwierigkeiten an der Universität

Robert W. Jahn

Stützlehrer als neuer pädagogischer Profi in der Beruflichen Bildung?! .... 147

Sabrina Berg

Pädagogische Praxis und Reproduktion sozialer Ungleichheit - zur

Berücksichtigung sozialer Herkunft im Wirtschaftsunterricht..... 161

\section{Teil V: Perspektiven der Berufsbildungsforschung}

\section{Miriam Voigt}

Neo-institutionalistische und mikropolitische Prozesse in

Schulentwicklungsprojekten

Lara Forsblom, Lucio Negrini, Jean-Luc Gurtner \& Stephan Schumann

Lehrvertragsauflösungen und die Rolle der betrieblichen Auswahl von

Auszubildenden 
Marius R. Busemeyer

Organisierte Interessen, Parteipolitik und institutioneller Wandel im deutschen Berufsbildungssystem

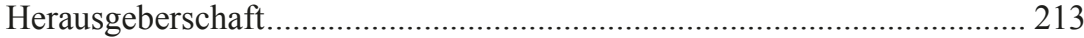

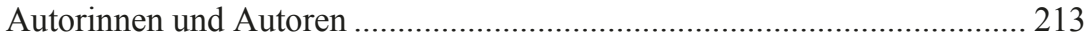




\title{
Möglichkeiten und Notwendigkeiten historiographischen Arbeitens in der Berufs- und Wirtschaftspädagogik
}

\author{
Frank-Lothar Kroll
}

Volker Bank zum 24. April 2014

\section{Gemeinsame Sinnhorizonte}

Wer es unternimmt, ${ }^{1}$ das Wechselverhältnis zweier in ihrer Wissenschaftsauffassung deutlich voneinander geschiedener Disziplinen zu bestimmen, der tut gut daran, sich zuvor über das ausgewiesene Selbstverständnis des jeweiligen Faches Klarheit zu verschaffen. Die Historiker streiten sich seit fast dreihundert Jahren über dieses Selbstverständnis - mal heftiger, mal weniger heftig, derzeit hält sich der Methodendiskurs eher in Grenzen. Man wird dabei zunächst, ganz allgemein, feststellen können, dass der Historiker die Erforschung von Vergangenem anhand der Ermittlung von Tatsachen betreibt, dadurch wird er zum Wissenschaftler (Wittram 1958, S. 55ff.). Denn Tatsachenermittlung, Tatsachenerkenntnis und Tatsachenpräsentation sind Kennzeichen aller empirischen Wissenschaften. Tatsachenbezug ist geradezu „der Ausweis der Wissenschaftlichkeit im Gegensatz zur bloßen Vermutung, zur unbewiesenen Behauptung, zum Ausdruck eines Wünschens und Wollens oder aber zur moralischen oder rechtlichen Bewertung" (Sellin 2001, S. 17). Allerdings verknüpft der Historiker die ihm zur Verfügung stehenden Tatsachen zu einem Sachzusammenhang, denn sonst wären sie weitgehend bedeutungslos. Mit diesem Verknüpfungsgeschäft verbindet sich wiederum ein Interpretationsvorgang, der Sinn stiftet und damit - unausweichlich - die Grenzen wissenschaftlicher Objektivität historiographischen Bemühens markiert.

Solche Sinnstiftung und Interpretation betreibt der Historiker nun freilich nicht als ein müßiges Geschäft im luftleeren Raum. Er hat ein Anliegen, das in seinen Einzelheiten vielfach variieren kann, auf dessen generelles Erkenntnisziel sich jedoch all sein Bestreben letztlich dauerhaft verpflichten lassen muss, wenn es denn Anspruch auf gesellschaftliche Relevanz und öffentliches Gehör erheben will. Dieses Grundanliegen allen historiographischen

1 Dieser Text ist die überarbeitete Fassung des Eröffnungsvortrags, den der Verfasser anlässlich der DGfE-Sektionstagung zur Berufs- und Wirtschaftspädagogik am 26. September 2013 in der Technischen Universität Chemnitz gehalten hat. Der Vortragsstil wurde beibehalten. 
Bemühens lautet, auf den einfachsten möglichen Nenner gebracht: intellektuelle Selbsterziehung, oder - wie es Karl Raimund Popper schon 1961 treffend formuliert hat: „Selbstbefreiung durch das Wissen“ (Popper 1961, S. 101). Jeder schulische Adept der Geschichte, jeder studentische Nachwuchshistoriker, jeder laienhaft interessierte Leser eines historischen Buches begegnet - im Idealfall - Sachverhalten und Sinnzusammenhängen, die ihm zur Erweiterung seines eigenen Horizontes verhelfen, weil sie eine Bereicherung der persönlichen Existenz durch verstehenden Nachvollzug einstmals getroffener menschlicher Entscheidungen im Beziehungsgeflecht gewesener Wirklichkeit ermöglichen. Eine ganze Welt erregender Erlebnisse eröffnet sich hier dem Rückblick des aufgeschlossenen Betrachters. Da gibt es Verfehlungen und Versäumnisse, die ihn betrüben, es gibt Größe, die ihn beeindruckt, Unglück das ihn ergreift, niederträchtiges Handeln, das ihn empört, Schuld, die ihn beschämt, doch auch Großmut und Noblesse, die ihn ermutigen. „Je mehr Vergangenheit wir überblicken, desto mehr erfahren wir vom Menschen" (Wittram 1958, S. 115) - und damit prinzipiell stets auch von uns selbst, von unserer Stellung in der Welt.

Dieses anthropologische Grundanliegen der Geschichtswissenschaft ist nicht unbedingt identisch mit dem Grundanliegen der Berufs- und Wirtschaftspädagogik. Aber es ist auch nicht allzu weit von ihm entfernt. Als fachfremder Dilettant mag man zunächst davon ausgehen, dass die Berufsund Wirtschaftspädagogik durch ein klar umrissenes fachliches Profil gekennzeichnet sei: Betriebliche und außerbetriebliche Weiterbildung; Qualifikationsprozesse an Beruflichen Schulen; Vermittlung praktischer Kompetenzen und grundlegenden Theoriewissens; Betreuung der im Berufs- und Wirtschaftsleben Stehenden zum Zweck ihrer personalen Selbstverwirklichung ebenso wie zur Erhöhung ihrer sozialen Aufstiegschancen und Entwicklungsmöglichkeiten - so etwa lauten für den Außenstehenden die zwar sehr allgemeinen, doch auf ein durchaus konsistentes Profil verweisenden Parameter berufs- und wirtschaftspädagogischen Selbstverständnisses.

Im näheren Zugriff auf einschlägige Handbücher (Rauner 2006) und Grundlagenliteratur (Kösel 2002, Zabeck 2013) stellt der fachferne Dilettant jedoch fest, dass innerhalb der Berufs- und Wirtschaftspädagogik die Frage nach dem fachspezifischen Erkenntnisinteresse keineswegs ohne weiteres zu beantworten ist; mehr noch: er muss mit einiger Überraschung konstatieren, dass die Berufs- und Wirtschaftspädagogik - nach den provozierenden Worten eines ihrer Hauptvertreter - ,gar keine gesicherte Disziplin“, sondern ein in hohem Maße ,disziplinloses Fach“ sei, welches, „kaum halbwegs etabliert, [schon] wieder [im Begriff stehe,] aus dem Lauf der Geschichte zu geraten“" (Bank 2007, S. 319, 325). Dies hänge zusammen mit der historisch auffallend spät erfolgten funktionalen Differenzierung des Faches innerhalb der Geisteswissenschaften, welche wiederum mit dessen nach wie vor schwankender inhaltlicher Selbstverortung, seinem Oszillieren zwischen verschiedenen 
Wissenskulturen zu erklären sei. Denn Berufs- und Wirtschaftspädagogik könne ,weder nur pädagogische noch nur wirtschaftswissenschaftliche Disziplin“ sein, gründe sich vielmehr ,auf die wechselseitigen instrumentellen Verhältnisse von Erziehung und Ökonomie, von Wirtschaften und Erziehen“ (Bank 2007, S. 324).

Solche Darlegungen fordern den nicht unmittelbar zum Fach zählenden Beobachter beinahe dazu heraus, sich mit einer derart in Grenzbereichen bewegenden und ganz offensichtlich auf Grenzüberschreitungen angewiesenen Disziplin zu beschäftigen und die dem Fach ausdrücklich zugesprochene „Anschlussfähigkeit“ (Bank 2007, S. 321) durch Brückenschläge zur eigenen Disziplin, der Geschichtswissenschaft, zu erproben.

\section{Geschichtsinteresse und Öffentlichkeit}

Einen nachvollziehbaren Ausgangspunkt für ein solches Unterfangen bietet die allseits sichtbare Präsenz historischer Bezüge in der modernen Lebenswelt. Seit mehreren Jahrzehnten floriert eine wahre "Gedächtniskultur" (Assmann 2012), die sich, unter anderem, in Ausstellungen und Memorialfeiern, in der Errichtung von Gedenkstätten und Mahnmälern und in der Rekonstruktion historischer Bauten (etwa der Dresdner Frauenkirche, des soeben fertiggestellten Potsdamer Stadtschlosses oder des gerade in Bau genommenen Berliner Schlosses) manifestiert. Auch die Erinnerung an historische Jubiläen (etwa den 100-jährigen Ausbruch des Ersten Weltkriegs 2014 oder die 250-jährige Beendigung der Sächsisch-polnischen Staatenunion 2013), juristische Prozesse und Gerichtsverfahren oder publikumswirksame Inszenierungen von "Geschichte“ in gedruckten oder elektronischen Medien bringen die fortdauernde ,gesellschaftliche Relevanz der Historie“ (Nipperdey 1972, S. 14) eindrucksvoll zum Ausdruck. Ob diese wahre Flut von erinnerungskulturellen Leistungen und Inszenierungen einem echten Geschichtsinteresse entspricht, ist allerdings nicht immer unbedingt ersichtlich. Fast immer werden die Modalitäten und Mechanismen der „Erinnerungskultur“ (Cornelissen 2003) von geschichtspolitischen Gesichtspunkten bestimmt, zumeist spielen auch kommerzielle Wünsche eine Rolle. Aber jedenfalls wird man bei alledem von einem „Verlust der Geschichte“, wie er noch in der alten Bundesrepublik vielfach beklagt worden ist (Heuss 1959, S. 5), heute nicht mehr ernsthaft sprechen können.

Die vielfältigen Formen kollektiven Erinnerns, an deren Gestaltung die Historiker zwar mitgewirkt haben, die im Verlauf des Memorialprozesses jedoch eine wachsende Eigendynamik entwickeln und sich konkreten korrigierenden Einflussmöglichkeiten der Zunft weitgehend entziehen, spielen auch für die Adepten und Adressaten der Berufs- und Wirtschaftspädagogik eine 
nicht unerhebliche Rolle. Gerade in der Wirtschaft fehlt es nicht an einer geschichtsbezogenen „Marketingisierung und Eventisierung“ der jeweiligen Unternehmenskommunikation (Grieger 2012, S. 96ff.). Fast täglich erscheinen raumfüllende Werbeanzeigen mit historischen oder pseudohistorischen Argumenten in den Medien. Firmenjubiläen geben darüber hinaus zahlreichen Unternehmen einen willkommenen Anlass, die Geschichte ihres Hauses durch ausgewiesene Historiker erarbeiten und publizieren zu lassen. Oftmals berühren solche Vorhaben, bei deren Realisierung pädagogisches Fachwissen und didaktisches Fingerspitzengefühl gefragt sind, äußerst sensible Problemstellungen - etwa hinsichtlich der Einbindung vieler deutscher Unternehmen in das Zwangsarbeitersystem des Dritten Reiches oder im Blick auf die aktive Beteiligung international renommierter deutscher Bankhäuser an antijüdischen Maßnahmen im Zusammenhang der als „Arisierung“ getarnten Beraubung fremden Eigentums. All das verlangt nach wissenschaftlicher Expertise, verbunden mit einem verantwortungsvollen Umgang der jeweiligen Unternehmen mit ihrer eigenen Geschichte. In den beiden letzten Jahrzehnten sind auf diesem Weg zahlreiche beachtenswerte Forschungsergebnisse zutage gefördert worden (Grieger 2007, Kukowski/Boch 2014). Der Steigerung des Absatzes entsprechend ,beworbener“ Produkte kam dies ebenso zugute wie der Festigung der Glaubwürdigkeit ihrer Produzenten.

Damit zusammenhängende Fragen und Problemstellungen mögen im aktuellen Lehrplan der Berufs- und Wirtschaftspädagogik eine nur sehr untergeordnete Rolle spielen. Aus der Sicht des Historikers wäre die Implementierung eines entsprechend ausgearbeiteten Moduls in den Ausbildungskanon dieses Studiengangs allemal sinnvoll. Die sich zusehends differenzierende öffentliche Geschichtskultur könnte vor den oftmals allzu dilettantisch betriebenen Bemühungen außerwissenschaftlicher Geschichtsbildproduzenten durch Präsentation einer curricularen Alternative jedenfalls potentiell einigermaßen geschützt werden.

Auf Seiten der universitären Geschichtswissenschaft sind in den letzten Jahren bereits Studienmodelle entwickelt worden, die in diese Richtung zielen. An der Freien Universität Berlin und an der Universität Heidelberg werden seit 2008 bzw. 2010 Masterstudiengänge zur Geschichtspraxis in „Public History“ angeboten. Dort soll - nach Ausweis der Studiendokumente - dem ,hohen medialen Interesse an Geschichte sowie der gewachsenen gesellschaftlichen Bedeutung von Museen, Gedenkstätten und anderen historischen Lernorten“ Rechnung getragen und dabei vor allem für solche Tätigkeiten qualifiziert werden, welche ,die Aufbereitung und Vermittlung fachwissenschaftlicher Erkenntnisse in einem breiten öffentlichen Kontext erfordern“ (Grieger 2012, S. 96). In ähnliche Richtungen zielt ein seit 2010 an der ETH Zürich aufgelegtes Masterprogramm für „Applied History“, das sich ausdrücklich als berufsbegleitender Studiengang ausweist. Im Sinne eines historischen Weiterbildungsangebots wendet er sich an bereits etablierte 
Praktiker der öffentlichen Geschichtsvermittlung - an Medienvertreter und Journalisten, an Manager und Verantwortliche in Verwaltung und Wirtschaft, ja sogar an Politiker, Dolmetscher und angehende Diplomaten, für die vertiefte historische Kenntnisse oftmals tatsächlich einen Weg zu verstärktem Erfolg und beruflichem Aufstieg ebnen mögen. Das Studium der Geschichte erfährt durch solche Modelle auf jeden Fall Bereicherung und Vervielfältigung, weil sie die akademische Ausbildung der Studierenden nicht mehr nur an rein wissenschaftlichen Kriterien orientieren, sondern Aspekten des Marktes und der Nachfrage ebenso entgegenkommen wie sie mögliche Erwerbsfelder und zukünftige Karrierechancen berücksichtigen.

\section{3. „Vergangenheitsbewirtschaftung“ als Bindeglied}

Die vielfältige Nutzanwendung von „Geschichte“ im öffentlichen Raum hat das Wirtschaftsleben mittlerweile in einem Ausmaß erfasst, wie es sich vor zwei Jahrzehnten nicht einmal die kühnsten Propheten und Prognostiker eines globalen Marktes erträumten (Kühberger 2012, S. 14ff.). Geschichte ist zu einem Wirtschaftsfaktor von erheblichem Rang geworden, Vergangenheit wird ,bewirtschaftet", das heißt systematisch vermarktet und für Werbezwecke eingesetzt. Solche „Vergangenheitsbewirtschaftung“ hat mehrere Zielgruppen im Blick. An erster Stelle rangieren hier die Kunden und Konsumenten. Ihre Käuferlaune soll inspiriert, ihr Kaufverhalten durch das Herausstellen bestimmter markenbezogener Merkmale nachhaltig aktiviert werden, indem auf „Tradition“, „Nostalgie“ oder „Hundertjährige Erfahrung“ und das damit verknüpfte Ansehen der jeweiligen Firma verwiesen wird. Vertraut man den Hoffnungen der Werbepsychologen und Marktstrategen, so gibt es derzeit kaum ein geeigneteres Mittel, um das Ansehen der jeweiligen Marke zu heben, ihren Absatz zu steigern, die erwarteten Gewinne zu maximieren und überhaupt Wettbewerbsvorteile großräumig zu verbessern. „History Marketing" gehört insofern in den Bereich allgemein ökonomischer Effizienzüberlegungen (Schug 2009, S. 21).

Doch dient die Aktivierung der eigenen Unternehmensvergangenheit nicht nur der werbenden Außendarstellung des Betriebes. Gerade marktführende, global vernetzte Firmen nutzen historisierende Versatzstücke zunehmend auch im Interesse einer positiven ,internen“ Sinnstiftung und betriebsbezogenen Identitätsformung. Ein derart nach innen gerichtetes „History Marketing“ (Bauer/Windischbauer 2012, S. 88) soll die Loyalität der Mitarbeiter stärken, ihr Identifikationspotential kanalisieren und ihr Handeln auf „altbewährte“ Firmenüberlieferungen verpflichten.

Auf diesem weiten Feld der „Geschichtsbewirtschaftung“, des „History Consulting“ und „History Management“ reichen oberflächlich erworbene Ge- 
schichtskenntnisse in der Regel nicht aus - vor allem dann nicht, wenn man „Geschichte“ nicht nur als singuläres Werbemittel zum Einsatz bringen will, sondern den Umgang mit Vergangenheit professionell für eine um Langfristigkeit und Nachhaltigkeit bemühte Unternehmenskultur zu nutzen beabsichtigt. Die Nachfrage entsprechender Dienstleistungen in diesem Umfeld generiert mittlerweile einen keineswegs mehr unbeträchtlichen Markt, der für die Ausbildungsziele des Faches „Berufs- und Wirtschaftspädagogik“ genauso attraktiv sein dürfte wie für die professionellen Platzhalter der Geschichtswissenschaft. All das macht die Vermittlung ,angewandter" Geschichtskenntnisse an die Studierenden benachbarter Disziplinen heute mehr denn je erforderlich. Daß dabei in der Regel ,ein freierer Umgang mit Geschichte möglich ist" (Lindner 2012, S. 63), als ihn tradierte akademische Standards und eingeübte Kriterien universitär verorteter Wissenschaftlichkeit gewöhnlich zulassen, hat der Historiker vom Fach nicht nur notgedrungen zu akzeptieren, sondern als Chance wahrzunehmen, neue Wege und Formen der Wissensvermittlung zu erproben, „die verstehbar, verständlich und plausibel“ sind (Schöllgen 2007, S. 11). Längst schon werden ja zahlreiche von ihm für richtig gehaltene Tatsachen und Wahrheiten von den Produzenten des beschriebenen „Memorybooms“ in interessengeleitete Engführungsprozesse eingespeist, in deren Einzugsfeld sie einer zweckgebundenen „Verwertung“ und „Vermarktung“ ebenso unterliegen (Nannen 2010, S. 8ff, 282ff.), wie sie in dieser spezifisch verwandelten Gestalt ihrerseits wiederum auf die Tätigkeit und die Sichtweise des Historikers zurückwirken.

\section{Ortsbestimmung in der Gegenwart}

Die bisherigen Darlegungen haben weit herangeführt an Grenzfragen des berufs- und wirtschaftspädagogischen Selbstverständnisses, soweit sich solche Fragen mit Anliegen und Arbeitsweisen des Historikers verknüpfen lassen. Wenn nun, abschließend, noch einmal auf das Ethos geschichtswissenschaftlichen Erkenntnisstrebens zurückzukommen ist, so geschieht auch dies in der Absicht, verwandte Strebungen zwischen beiden Disziplinen aufzudecken.

Allen skizzierten Formen professioneller „Vergangenheitsbewirtschaftung“ liegt ein ausnahmslos instrumentelles Interesse an historischen Sachverhalten zugrunde. Sofern die damit verknüpften Ziele nicht mit den Mitteln der Willkür oder gar der bewußten Verfälschung zu realisieren versucht werden, unterscheiden sie sich vom Bildungsanliegen der wissenschaftlichen Historiographie nur graduell, nicht jedoch prinzipiell. Denn auch die Aktivitäten des Historikers sind grundsätzlich immer praxisbezogen - betrieben in einem aristotelischen Verständnis von „Praxis“ als Dienerin und Wegbereiterin eines guten und sinnvoll gelebten Lebens auf der Höhe der Zeit. Lebens- 
praktischen Wert besitzt die Historie ja nicht nur im begründeten Zurückweisen schädlicher und verhängnisvoller Weltdeutungen von rechts oder von links. Geschichtswissen ermöglicht darüber hinaus in zahlreichen politisch und gesellschaftlich relevanten Fragen überhaupt erst eine adäquate Ortsbestimmung, und es gehört zu den Übelständen der Gegenwart, daß über solche Fragen nur allzu oft in vollendeter Unkenntnis historischer Zusammenhänge diskutiert wird. Wer in historischen Dimensionen denkt, dem eröffnen sich intellektuelle Einsichten und praktische Handlungsoptionen, die den mittleren Horizont bloßer Tagesgesichtspunkte weit überragen.

Auch zum Sammeln lehrreicher Erfahrungen bietet der Blick in die Geschichte mancherlei willkommene Fingerzeige. Hegel hatte das in seiner „Philosophie der Weltgeschichte“ bekanntlich mit dem Verweis darauf bestritten, „daß Völker und Regierungen niemals etwas aus der Geschichte gelernt und nach Lehren, die aus derselben zu ziehen gewesen wären, gehandelt haben“ (Hegel 1970, S. 17). Das ist richtig und falsch zugleich: Selbstverständlich kann ein „Lernen aus der Geschichte“ nicht in Form naiver Übertragbarkeiten oder Rezeptanweisungen vom „Einst" ins „Jetzt" erfolgen. Aber Möglichkeiten des Handelns lassen sich durch eine dosierte Rücksichtnahme auf historisches Erfahrungswissen sehr wohl schärfer profilieren und sei es nur, um zu erkennen, daß keine Geschichtskonstellation unumkehrbar ist oder gar ,alternativlos" daherkommt.

„Selbstbefreiung durch das Wissen“ - darin hatte Karl Raimund Popper die anthropologische Zielvorgabe historiographischen Arbeitens erblickt und damit, wenn man so will, eine Möglichkeit des Brückenschlags zu verwandten Tendenzen innerhalb des Faches „Berufs- und Wirtschaftspädagogik“ eröffnet, wie er hier versucht worden ist. Das Studium der Geschichte, so kann man Poppers Dictum weiterführend kommentieren, formt Haltungen und Einstellungen, „die einem Leben in Gemeinschaft jedenfalls nicht hinderlich, für die eigene Daseinsbewältigung jedoch mit Sicherheit hilfreich sein dürften“ (Sellin 2001, S. 209). Wilhelm Busch, der Meister gepflegtsarkastischer Ironie, hat dieses anthropologische Grundanliegen der Beschäftigung mit Geschichte und der Beschaffung historischen Wissens in seiner Gedichtsammlung „Kritik des Herzens“ mit unübertroffener Prägnanz zu formulieren gewußt: „Früher, da ich unerfahren/und bescheidner war als heute/hatten meine höchste Achtung/andere Leute. //Später traf ich auf der Weide/außer mir noch mehre Kälber,/und nun schätz' ich, sozusagen,/erst mich selber" (Busch 1958, S. 248). 


\section{Literatur}

Assmann, A. (2012): Auf dem Weg zu einer europäischen Gedächtniskultur? Wien: Picus.

Bank, V. (2007): Berufs- und Wirtschaftspädagogik: Epitaph einer Disziplinlosen. In: Wirtschaft und Erziehung 59. Bd., S. 319-330.

Bauer, J./Windischbauer, E. (2012): History Marketing als Strategie einer nach innen gerichteten Unternehmenskommunikation. In: Kühberger, C./Pudlat, A. (Hrsg.): Vergangenheitsbewirtschaftung. Public History zwischen Wirtschaft und Wissenschaft. Innsbruck/Wien/Bozen: Studien Verlag, S. 88-95.

Bösch, F./Goschler, C. (Hrsg.) (2009): Public History. Öffentliche Darstellungen des Nationalsozialismus jenseits der Geschichtswissenschaft. Frankfurt am Main/ New York: Campus.

Cornelissen, C. (2003): Was heißt Erinnerungskultur? Begriff - Perspektiven. In: Geschichte in Wissenschaft und Unterricht 54. Bd., S. 548-563.

Busch, W. (1958): Kritik des Herzens. Gesamtausgabe, Bd. 5. München.

Grieger, M. (2012): Zur Hybridisierung der Unternehmensgeschichte durch Verwissenschaftlichung, Marketingisierung und Eventisierung. Das Beispiel Volkswagen. In: Kühberger, C./Pudlat, A. (Hrsg.): Vergangenheitsbewirtschaftung. Public History zwischen Wirtschaft und Wissenschaft. Innsbruck/Wien/Bozen: Studien Verlag, S. 96-119.

Hegel, G. W. F. (1970): Vorlesungen über die Philosophie der Geschichte. Werke, Bd. 12. Frankfurt am Main: Suhrkamp.

Heuss, A. (1959): Verlust der Geschichte. Göttingen: Vandenhoeck \& Ruprecht.

Kösel, E. (2002): Die Modellierung von Lernwelten. Bd. I: Die Theorie der Subjektiven Didaktik. Wissenschaftliche Grundlagen. 4. Aufl. Bahlingen: Kösel.

Kühberger, C. (2012): Geschichtsmarketing als Teil der Public History. Einführende Sondierungen zwischen Wissenschaft und Wirtschaft. In: Kühberger, C./Pudlat, A. (Hrsg.): Vergangenheitsbewirtschaftung. Public History zwischen Wirtschaft und Wissenschaft. Innsbruck/Wien/Bozen: Studien Verlag, S. 44-53.

Kühberger, C. (2009): Kompetenzorientiertes historisches und politisches Lernen. Methodische und didaktische Annäherungen für Geschichte, Sozialkunde und Politische Bildung. Innsbruck/Wien: Studien Verlag.

Kühberger, C. (2009): Verkaufte Zunft? Ein Beitrag zur Ethik des History Consulting. In: Hardtwig, W./Schug, A. (Hrsg.): History sells! Angewandte Geschichte als Wissenschaft und Markt. Stuttgart: Steiner, S. 43-53.

Kühberger, C./Lübke, C./Terberger, T. (Hrsg.) (2007): Wahre Geschichte - Geschichte als Ware. Die Verantwortung der historischen Forschung für Wissenschaft und Gesellschaft. Rahden/Westf.: VML.

Kukowski, M./Boch, R. (2014): Kriegswirtschaft und Arbeitseinsatz bei der Auto Union AG Chemnitz im Zweiten Weltkrieg. Stuttgart: Steiner.

Lindner, J. (2012): „Wir machen hier Marketing und keine Wissenschaft“. Akteure und ihre Handlungsspielräume am Beispiel des „History Marketing“. In: Kühberger, C./Pudlat, A. (Hrsg.): Vergangenheitsbewirtschaftung. Public History zwischen Wirtschaft und Wissenschaft. Innsbruck/Wien/Bozen: Studien Verlag, S. 54-69. 
Nannen, K. (2010): Wirtschaft, Geschichte und Geschichtskultur. Eine Untersuchung zur Vermarktung und Förderung von Geschichte durch deutsche Unternehmen. Münster: Lit.

Nipperdey, T. (1976): Über Relevanz (1972). Wiederabgedruckt in: Ders.: Gesellschaft, Kultur, Theorie. Gesammelte Aufsätze zur Neueren Geschichte. Göttingen: Vandenhoeck \& Ruprecht, S. 12-32.

Popper, K. R. (1961): Selbstbefreiung durch das Wissen. In: Reinisch, L. (Hrsg.): Der Sinn der Geschichte. Sieben Essays. München: Beck, S. 100-116.

Rauner, F. (Hrsg.) (2006): Handbuch der Berufsbildungsforschung. Bielefeld: WBV.

Schöllgen, G. (2007): Die Dienstleister. Von den Aufgaben der Geisteswissenschaften in der modernen Welt. Erlangen: Friedrich-Alexander-Universität ErlangenNürnberg.

Schug, A. (2003): History Marketing. Ein Leitfaden zum Umgang mit Geschichte in Unternehmen. Bielefeld: Transcript.

Sellin, V. (2001): Einführung in die Geschichtswissenschaft (zweite., durchges. Aufl.). Göttingen: Vandenhoeck \& Ruprecht.

Wittram, R. (1958): Das Interesse an der Geschichte. Zwölf Vorlesungen über Fragen des zeitgenössischen Geschichtsverständnisses. Göttingen: Vandenhoeck \& Ruprecht.

Wittram, R. (1958): Das Faktum und der Mensch. Bemerkungen zu einigen Grundfragen des Geschichtsinteresses. In: Historische Zeitschrift 185. Bd., S. 55-87.

Zabeck, J. (2013): Geschichte der Berufserziehung und ihrer Theorie (zweite, erw. und überarb. Aufl.). Paderborn: Eusl. 